\title{
Expression of Wnt Genes in Echinaster Sepositus Arm Regeneration
}

\author{
Ben Khadra $\mathrm{Y}^{1 *}$, Dimassi $\mathrm{N}^{1}$ and Martinez $\mathrm{P}^{2,3}$ \\ ${ }^{1}$ Laboratory of Genetics, Biodiversity and Valorization of Bioresources, Higher \\ Institute of Biotechnology, University of Monastir, Tunisia \\ 2Institut Català de Recercai Estudis Avancats (ICREA), Spain \\ ${ }^{3}$ Genetics Department, University of Barcelona, Spain
}

\section{Research Article \\ Volume 3 Issue 1}

Received Date: December 27, 2017

Published Date: January 24, 2018

*Corresponding author: Yousra Ben Khadra, Laboratory of Genetics and biodiversity, University of Monastir, Rue Tahar Haddad, 5000, Monastir, Tunisia, Tel: 002165871225; E-mail: youssra_benkhadra@yahoo.fr

\section{Abstract}

Reparative regeneration is defined as the replacement of lost adult body parts and is a phenomenon widespread yet highly variable among animals, and thus thought to have evolved several times. This raises the question of which key molecular mechanisms have to be implemented in order to efficiently and correctly replace entire body parts. Although arm regeneration in Asteroids is a quite-well studied phenomenon, numerous important aspects have still to be defined in terms of molecular mechanisms. The present work is focused on the expression of Wnt genes in the red starfish Echinaster sepositus (Asteroidea, Echinodermata) arm regeneration. A major reason for undertaking this study is that most of these genes have been previously associated with wound healing, development and regeneration in other animal species. Here, two new Wnt genes were characterized; EsWnt1 and EsWnt4. The expression profiles of these two genes using RT-PCR showed a temporal correlation with the known cellular events. An early expression of the EsWnt1 and EsWnt4 genes was noted after 24 hours of amputation. The number of transcripts of the EsWnt4 gene decreased considerably after 72 hours and disappeared completely by the first week. Later on we observed high levels after 7 weeks of amputation. A late expression of the EsWnt1 gene was seen, at the 4th week, and remaining at low levels until the 7 th week. In comparison with other organisms, the early expression of both genes suggests and involvement in wound healing and cell migration and, probably, also in the myogenesis and the establishment of antero-posterior polarity. These expression profiles support the hypothesis of the key regulatory functions of Wnt signalling pathways during embryogenesis and cellular renewal in adult regeneration.

Keywords: Regeneration; Sea star; Echinaster sepositus; Wnt1; Wnt4; Expression

\section{Abbreviations: AA: Amino Acid; PA: Post amputation}

\section{Introduction}

Regeneration is defined as a complete restoration of the morphology (anatomy) and the functions of damaged organs/tissues. Regeneration has been considered as an ancestral phenomenon that has been lost in many clades throughout evolutionary time; the capacity of regeneration seems to decrease with the complexity of organisms, from the most basal to the more derived taxa [1]. Moreover, it has been suggested that the lost of regeneration's ability is related to the 


\section{International Journal of Biochemistry \& Physiology}

evolutionary transition leading to the "exit of animals from the water environment" [2]. This would suggest that the marine invertebrates (such as echinoderms) maintain some of the ancestral mechanisms of regeneration.

The choice of the model organism in regeneration studies is fundamental, but tricky. The selection criteria could be made according to our interest for understanding the limited ability of vertebrates for regeneration, a biomedical interest, and this choice would depend, hence, of: (i) the phylogenetic relationship of our model with respect to the vertebrates and, obviously, of (ii) its regenerative potential. Thereby, echinoderms are excellent models for studying organ regeneration, since on the one side they can regenerate many of their organs and appendages [3], and on the other they represent a basal group of non-chordate deuterostomes, occupying a key position for understanding the evolution of vertebrates. In particular, several papers have documented that asteroids are outstanding examples of appendage regeneration within Echinodermata [4-6]. The regenerative capacities in sea stars have been mainly studied in the context of cell recruitment, dedifferentiation, proliferation and migration [4-8]. The cellular mechanisms of arm regeneration in different sea star species have been well documented [4-8], for instance (especially relevant here) in the Mediterranean red sea star Echinaster sepositus (Asteroidea, Echinodermata) [7,8]. The overall process of arm regeneration in E. sepositus can be subdivided into three main phases: a first "Repair phase: closure of the coelomic canals, re-epithelialisation and an oedematous area formation" (0 -1week post amputation (pa)), a second "Early regenerative phase: stump tissue rearrangement (mainly muscle bundles/layers)" (1 - 6 week(s) pa), and a third "Advanced regenerative phase: including skeletogenesis, myogenesis and neural differentiation" (from 6 weeks pa) [7,8]. Although, these studies succeeded at determining the detailed morphological aspects linked to arm regeneration of this asteroid, there is still a considerable gap in our knowledge of all molecular aspects regulating the process.

A key aspect that needs to be clarified is how the cells involved in the regenerating arm of E. sepositus must be arranged in the proper spatial positions during their proliferation and migration in order to form a complete and well patterned arm-tip. For precise cell positioning, these patterning signals are used to "guide" all implicated cells. The understanding of how arm regeneration polarity in sea stars is established, which requires the involvement of intrinsic regulative properties of the regenerating tissues, provides a paradigm for the study the process of tissue specification during regeneration. Since Homebox genes function as regulatory transcription factors and play important roles in metazoan patterning during development and in the regrowth of body parts after injury [9-11], an initial, comprehensive, Hox gene expression study was carried out in E. sepositus arm regeneration [6]. In this study we are interested in another set of positional regulators, and these are the members of the wingless/Wnt family. Wnts are a family of secreted glycoproteins with significant roles in many biological processes. Beside the crucial role of Wnt genes during early embryonic developmental processes, it has been demonstrated that Wnt signalling is also required for adult tissue regeneration in different species [12-14]. For example, blocking Wnt signalling disrupts the polarity of the regenerate in planarians, resulting in inappropriate anatomical architecture [1519]; and also impairs the normal fin regeneration in the teleost zebrafish [20]. Interestingly, Wht signalling is also necessary for the repair process in organisms with limited regenerative capacities (i.e.: mammalian, for review see [21]. While Wnt signalling pathways have been studied in different taxa, with robust or limited regenerative capacities, they have been rarely investigated in echinoderms. Only the expression of Wnt-9 homologous gene during intestine regeneration in the sea cucumber Holothuria glaberrima has been studied [22].

In this report, we describe the characterization of two Wnt genes expressed in the E. sepositus regenerating arm; the orthologous of Wnt1 and Wnt4 genes. The expression profiles of both genes at different stages of this sea star arm regeneration have been also studied. The obtained results corroborate, by comparison with the expression profiles of Wnt1 and Wnt4 in different invertebrate and vertebrate species, the involvement of Wnt signalling pathways in the regeneration of animals.

\section{Materials and Methods}

\section{Ethics statement}

All animal manipulations were performed according to the Tunisian law, i.e. no specific permits were required for the described studies as starfish are invertebrates. Echinaster sepositus is not an endangered or protected species. All efforts were made to minimize the animal suffering during experimental procedures. The specimens were released into their natural environment once the experimental procedures were completed.

\section{Animal Collection and Induction of Regeneration}

Adult (diameter $\sim 12 \mathrm{~cm}$ ) specimens of E. sepositus were collected by scuba divers from at the National Institute of Marine Sciences and Technologies of 


\section{International Journal of Biochemistry \& Physiology}

Monastir (INSTM, Tunisia) at depth of 5-8 m. To eliminate stress effects, sea star specimens were maintained at $16^{\circ} \mathrm{C}$ in a natural circulating seawater system for several days prior to the experiments. Specimens were fed with small pieces of cuttlefish twice a week. Traumatic amputation of the distal third of one arm for each specimen was performed by scalpel. Animals were then left to regenerate in the tanks for predetermined periods. The regeneration pattern was monitored at 1, 24, 72 hour(s), 1, 2, 3, 4 and 7 week(s) post-amputation (pa) and was also compared with that of normal non-regenerating arms ( 0 hour pa). The regenerating tissue samples were later collected at different stages and stored in RNA later (Sigma) for molecular analyses.

\section{RNA Isolation, cDNA Synthesis, PCR Amplification, and Cloning}

Total RNA was isolated from regenerating arm tips of E. sepositus using a miRCURY RNA Isolation Kit-Tissue (Exiqon). Each extract of RNA was treated with DNase (RNase free) (Invitrogen) to avoid any contamination with genomic DNA during amplification. The reverse transcriptase-polymerase chain reaction was used to detect wingless genes transcripts present in the regenerating tissues at different stages postamputation. The first-strand cDNA was synthesized by adding total RNA to a reaction mixture containing $10 \mathrm{X}$ RT buffer, $2 \mathrm{mM}$ DTT, $1 \mathrm{mM}$ dNTP mix, $1 \mu \mathrm{M}$ oligo dT primer, and $20 \mathrm{U}$ M-MLV reverse transcriptase, and then incubated at $42^{\circ} \mathrm{C}$ for $1 \mathrm{~h}$. Two oligonucleotides were used to amplify Wnt (Wnt-F and Wnt-R (Table 1)) as designed by Ferkowicz, et al. [23]. A second set of primers, ActF and ActR, derived respectively from the conserved peptide DGQVITIG and TFQQMWI, was used to amplify an Actin fragment, used later as an internal control in the RT-PCR. We performed 35 PCR cycles at $94^{\circ} \mathrm{C}$ for $1 \mathrm{~min}, 1 \mathrm{~min}$ at $55^{\circ} \mathrm{C}$ for Wnt genes and $50^{\circ} \mathrm{C}$ for Actin gene, $72^{\circ} \mathrm{C}$ for $2 \mathrm{~min}$, plus a final extension of $10 \mathrm{~min}$ at $72^{\circ} \mathrm{C}$. The PCR products were gel purified (QIAquick Gel Extraction Kit; Qiagen) and cloned into the pCR 2.1 vector (Invitrogen). White colonies were screened for the presence of inserts by PCR amplification using M13 universal and reverse primers. The insert-containing clones were then purified and sequenced using an automatic $\mathrm{ABI} 3700$ sequencer.

\begin{tabular}{|c|c|}
\hline Primer & Sequence (5' ${ }^{\prime} \mathbf{3}^{\prime}$ ) \\
\hline Act-F & 5'-AYGGWCAGGTYATCACCRTT-3' \\
\hline Act-R & 5'-ATCCACATCTGYTGGAAGGK-3' \\
\hline Wnt-F & 5'-GGGAATTCCARGARTGYAARTGYCAT-3' \\
\hline Wnt-R & 5'-AAAATCTAGARCARCACCARTGRAA-3' \\
\hline EsWX-F & 5'-TACGGAAAACAATTTCCAAC-3' \\
\hline EsWX-R & 5'-GACTTGCGAGCTATCGTAGT-3' \\
\hline EsWY-F & 5'-ACATCTAAGGTTTGCTGGAA-3' \\
\hline EsWY-R & 5'-GCAGAGTCTACCGTGAGTTC-3' \\
\hline EsAct-F & 5'-ATGGACAGGTCATCACCGTT-3' \\
\hline EsAct-R & 5'-ACCTTCCAGCAGATGTGGAT-3' \\
\hline
\end{tabular}

Table 1: Oligonucleotide primers used in this study

\section{Phylogenetic analysis}

Proteins sequences of Wht -related genes from different animals were obtained from GenBank (http://www.ncbi.nlm.nih.gov/Genbank/index.html)

[24]. Additional genomic data for the sea urchin Strongylocentrotus purpuratus, sequences SpWnt9 and SpWnt10 here, were obtained from BCM-HGSC [Baylor College of Medicine-Human Genome Sequencing Center] (http://www.hgsc.bcm.tmc.edu/blast/blast.cgi?organis $\mathrm{m}=$ Spurpuratus) (Table 2).
Proteins alignment was conducted using the default settings of the ClustalW program [25], implemented in MEGA v6.06 [26] and trimmed manually to fit Protein fragments length of both EsWntX and EsWntY sequences.

A neighbour-joining tree was generated using MEGA v6.06 [26], with 5000 bootstrap replicates. The evolutionary distances were computed using the pdistance method [27] and were given in the units of the number of amino acid differences per site. 


\section{International Journal of Biochemistry \& Physiology}

\begin{tabular}{|c|c|c|c|}
\hline Subfamily gene & Sequence Name & Accession Number & Species/Group of animal used \\
\hline \multirow{2}{*}{ EsWnt } & EsWntX & AGQ47097 & Echinoderm (Echinastersepositus) \\
\hline & EsWntY & AGQ47098 & Echinoderm (Echinastersepositus) \\
\hline \multirow{3}{*}{ Wnt1 } & HsWnt1 & NP_005421 & Vertebrate (Human) \\
\hline & EtWnt1 & P28089 & Echinoderm (Evasteriastroschelii) \\
\hline & SpWnt1 & NP_001116972 & Echinoderm (Strongylocentrotuspurpuratus) \\
\hline Wnt2 & NvWnt2 & AAW28132 & Cnidaria (Sea anemone) \\
\hline \multirow{3}{*}{ Wnt3 } & HsWnt3 & NP_110380 & Vertebrate (Human) \\
\hline & NvWnt3 & DQ492689 & Cnidaria (Sea anemone) \\
\hline & SpWnt3 & XP_790595 & Echinoderm (Strongylocentrotuspurpuratus) \\
\hline \multirow{3}{*}{ Wnt4 } & PlWnt4 & AHY22364 & Echinoderm (Paracentrotuslividus) \\
\hline & PmWnt4 & XP_014365310 & Insecta (Papiliomachaon) \\
\hline & SpWnt4 & XP_797603 & Echinoderm (Strongylocentrotuspurpuratus) \\
\hline \multirow{2}{*}{ Wnt5 } & SpWnt5b & XP_011670176 & Echinoderm (Strongylocentrotuspurpuratus) \\
\hline & SpWnt5 & AAC69436 & Echinoderm (Strongylocentrotuspurpuratus) \\
\hline \multirow{2}{*}{ Wnt6 } & NvWnt6 & AAW28134 & Cnidaria (Sea anemone) \\
\hline & SpWnt6 & XP_790077 & Echinoderm (Strongylocentrotuspurpuratus) \\
\hline \multirow{3}{*}{ Wnt7 } & NvWnt7b & AAW28135 & Cnidaria (Sea anemone) \\
\hline & NvWnt7 & AAV87176 & Cnidaria (Sea anemone) \\
\hline & SpWnt7 & AAA30087 & Echinoderm (Strongylocentrotuspurpuratus) \\
\hline \multirow{3}{*}{ Wnt8 } & HsWnt8B & NP_003384 & Vertebrate (Human) \\
\hline & NvWnt8b & AAW28136 & Cnidaria (Sea anemone) \\
\hline & SpWnt8 & NP_999832 & Echinoderm (Strongylocentrotuspurpuratus) \\
\hline \multirow{3}{*}{ Wnt9 } & HsWnt9A & NP_003386 & Vertebrate (Human) \\
\hline & HsWnt9B & NP_003387 & Vertebrate (Human) \\
\hline & SpWnt9 & [32] & Echinoderm (Strongylocentrotuspurpuratus) \\
\hline \multirow{2}{*}{ Wnt10 } & NvWnt10 & AAT00641 & Cnidaria (Sea anemone) \\
\hline & SpWnt10 & [32] & Echinoderm (Strongylocentrotuspurpuratus) \\
\hline Wnt11 & NvWnt11 & AAV87175 & Cnidaria (Sea anemone) \\
\hline \multirow{3}{*}{ Wnt16 } & HsWnt16 & NP_476509 & Vertebrate (Human) \\
\hline & NvWnt16 & DQ492688 & Cnidaria (Sea anemone) \\
\hline & SpWnt16 & XP_796616 & Echinoderm (Strongylocentrotuspurpuratus) \\
\hline \multirow{2}{*}{ WntA } & PdWntA & CAD37169 & Lophotrochozoans (Annelid) \\
\hline & PvWnyA & CAD37173 & Lophotrochozoans (mollusc) \\
\hline
\end{tabular}

Table 2: Wnt sequences used in the phylogenetic analysis

\section{Reverse Transcription Polymerase Chain Reaction (RT-PCR)}

In order to study the temporal expression of obtained Wnt genes, we performed RT-PCR to verify the presence or absence of the transcripts in the normal and regenerating arms at 1, 24, 72 hour(s), 1, 2, 3, 4 and 7 week(s) post-amputation (pa). Obtaining relevant results is dependent to the quantity and quality of total RNA used in the reverse transcription step. It is important to well quantify and qualify the extracts of RNA before cDNAs synthesis. All RNA extracts of each stage were analysed using the Agilent Bioanalyzer at the
"Transcriptomic Unit" of the scientific Park of the University of Barcelona. Based on the values of the RIN (RNA Integrity Number) of each extract we have chosen the purest samples of RNAs. Equal quantities of RNA of each extract $(2 \mu \mathrm{g})$ have been used in the synthesis of cDNAs using the kit $1^{\text {st }}$ Strand cDNA Synthesis Kit for RT - PCR (AMV) and following the instructions of the producer (Roche).Amplifications were carried out to amplify the fragments ( $200 \mathrm{bp})$ of WntX, WntY and Actin genes with new gene-specific primers ((EsWX-R, EsWX-F; EsWY-R, EsWY-F; EsAct-F and EsAct-R, Table 1).PCR products were analyzed by conventional agarose gel electrophoresis. 


\section{International Journal of Biochemistry \& Physiology}

\section{Results}

\section{Cloning of Wnt Genes in E. sepositus and Phylogenetic Analysis}

The degenerated primers, Wnt- $F$ and Wnt- $R$, allowed the amplification ofa fragment of $438 \mathrm{bp}$ of the genes EsWntX and a fragment of $355 \mathrm{bp}$ of gene EsWntY (temporary names) in the sea star E. sepositus. Similarly, using degenerated primers a fragment of $300 \mathrm{bp}$ of belonging to the Actin gene was amplified. The latter one used as a control for RT-PCR.

The identification of the identity of the EsWntX and EsWntY genes was determined by a phylogenetic analysis, using representative Wnt sequences from echinoderm, cnidarian, insect, mollusc, annelid and vertebrate lineages as references (Table 2). Wnt amino acid sequences were aligned and trimmed to a total of 174 aa. Amino acids alignment revealed that EsWntX and EsWntY possess a highly conserved amino acid sequence, when compared with the different Wnt members (Figure 1). The neighbour-joining tree based on 33 different species' Wnt sequences is shown in figure 2.

Phylogenetic analysis showed that EsWntX and EsWntY clustered, respectively, with the related species' Wnt1 and Wnt4 sequences (high bootstrap values). More specifically, it shows that EsWntX and EsWntY have the highest affinities with the members of the echinoderm orthologous families, as demonstrated by the high bootstraps obtained with the sequence of Evasterias troschelii Wnt1 (99\%) and with the sequence of the S. purpuratus Wnt4 genes (96\%).Moreover, and based on Blast analysis we concluded that the identified Actin fragment correspond to Actin1. After the phylogenetic analysis these fragments were all renamed, using a prefix indicating the derived species (EsE. sepositus) followed by a name that indicates the putative family to which they belong, thus: EsWnt1, EsWnt4 and EsActin1. These three newly characterized genes, EsWnt1, EsWnt4 and EsActin1, were submitted to the NCBI database, respectively, with the accession numbers: KC820638, KC820639 and KC858258.

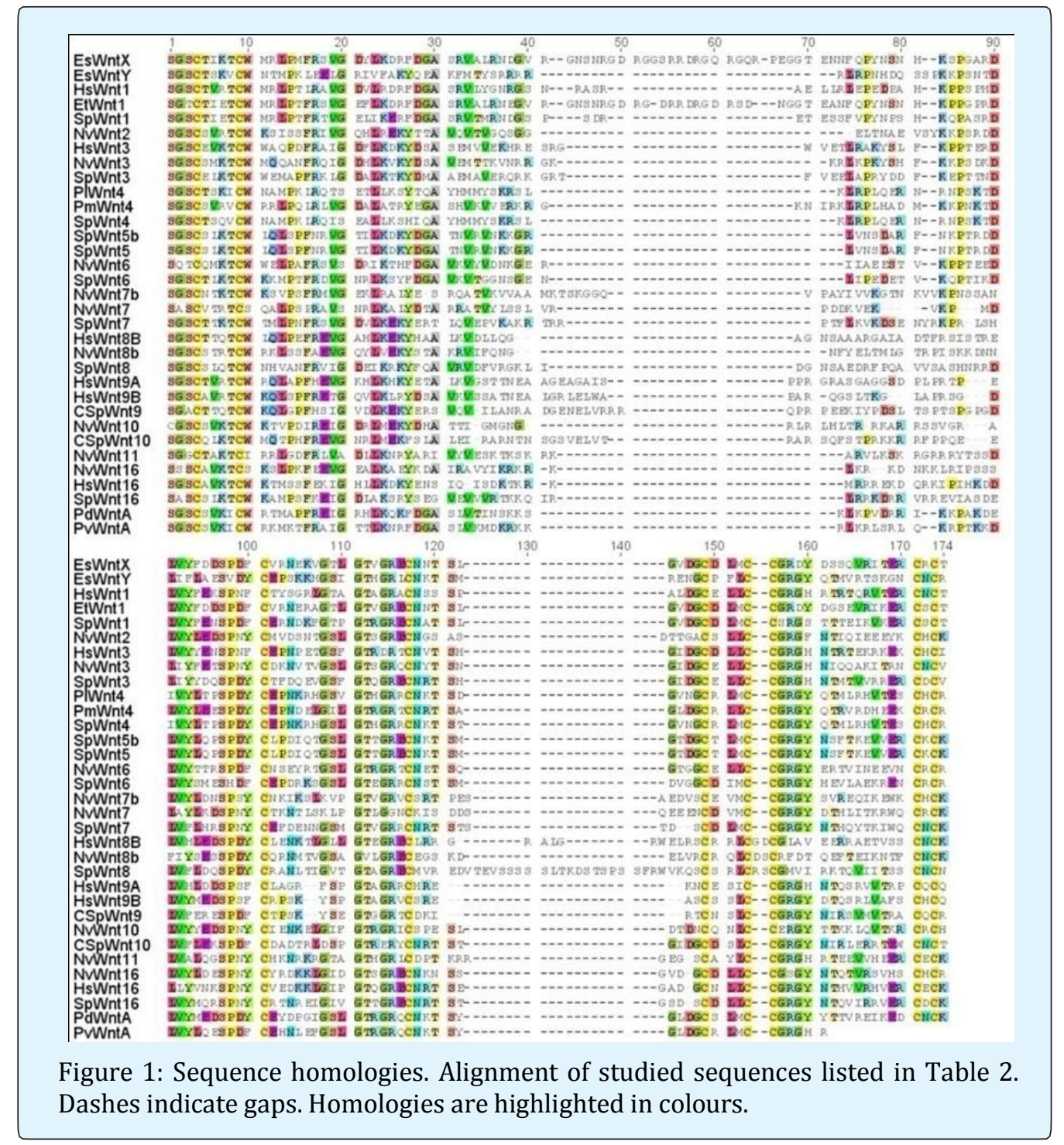




\section{International Journal of Biochemistry \& Physiology}

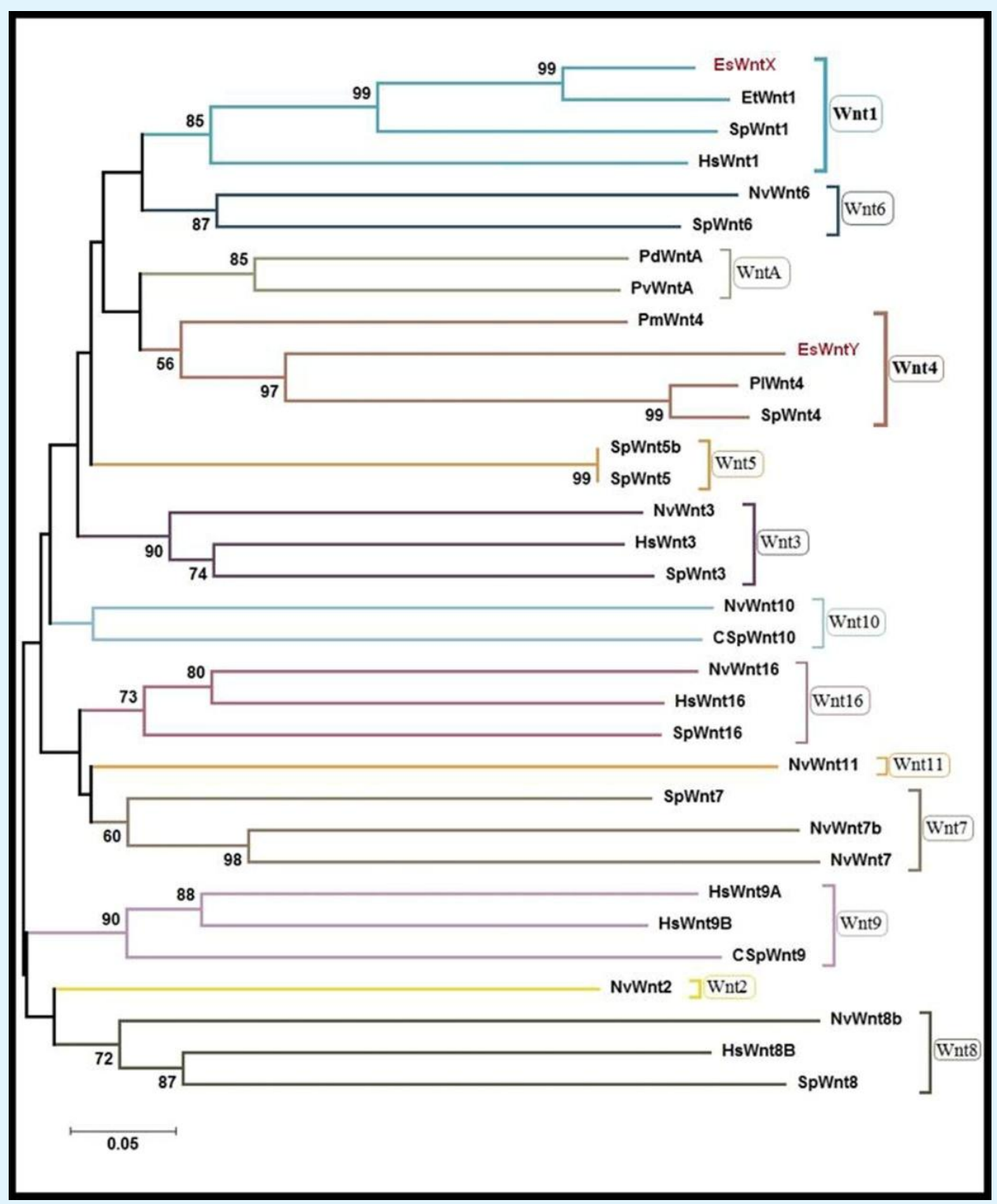

Figure 2: The evolutionary history was inferred using the Neighbor-Joining method. The optimal tree with the sum of branch length $=7.96635797$ is shown. The percentage of replicate trees in which the associated taxa clustered together in the bootstrap test (5000 replicates) are shown next to the branches. The tree is drawn to scale, with branch lengths in the same units as those of the evolutionary distances used to infer the phylogenetic tree. The evolutionary distances were computed using the p-distance method and are in the units of the number of amino acid differences per site. The analysis involved 33 amino acid sequences. All ambiguous positions were removed for each sequence pair. There were a total of 174 positions in the final dataset. Evolutionary analyses were conducted in MEGA6. Percentage of support (50\% or higher) in bootsrap searches is shown on the branches.

\section{Expression Profiles of EsWnt1 and EsWnt4}

The analysis of the temporal regulation of EsWnt1 (Figure 3B) and EsWnt4 (Figure 3C) genes showed variations in their expression levels through the different stages of regeneration. This expression was normalized to the expression of a housekeeping gene EsActin1, which appeared invariable at all stages of regeneration (Figure $3 \mathrm{~A}$ ). In non-amputated arms (0 hour pa) the expression of these two Wnt genes was zero (below detection levels). An early period of expression of EsWnt1 and EsWnt4 genes was noted after 24 hours of the amputation. The number of transcripts of EsWnt4 gene decreased noticeably after 72 hours and disappeared completely at the end of the $1^{\text {st }}$ week to increase again during the period leading to 7 weeks after the amputation. Contrary to EsWnt4, the expression of EsWnt1 gene increased at 72 hours pa compared to the previous stage ( 24 hours pa). A late expression of EsWnt1 gene was detected at the $4^{\text {th }}$ week and the expression levels remained stable until the $7^{\text {th }}$ week. 


\section{International Journal of Biochemistry \& Physiology}

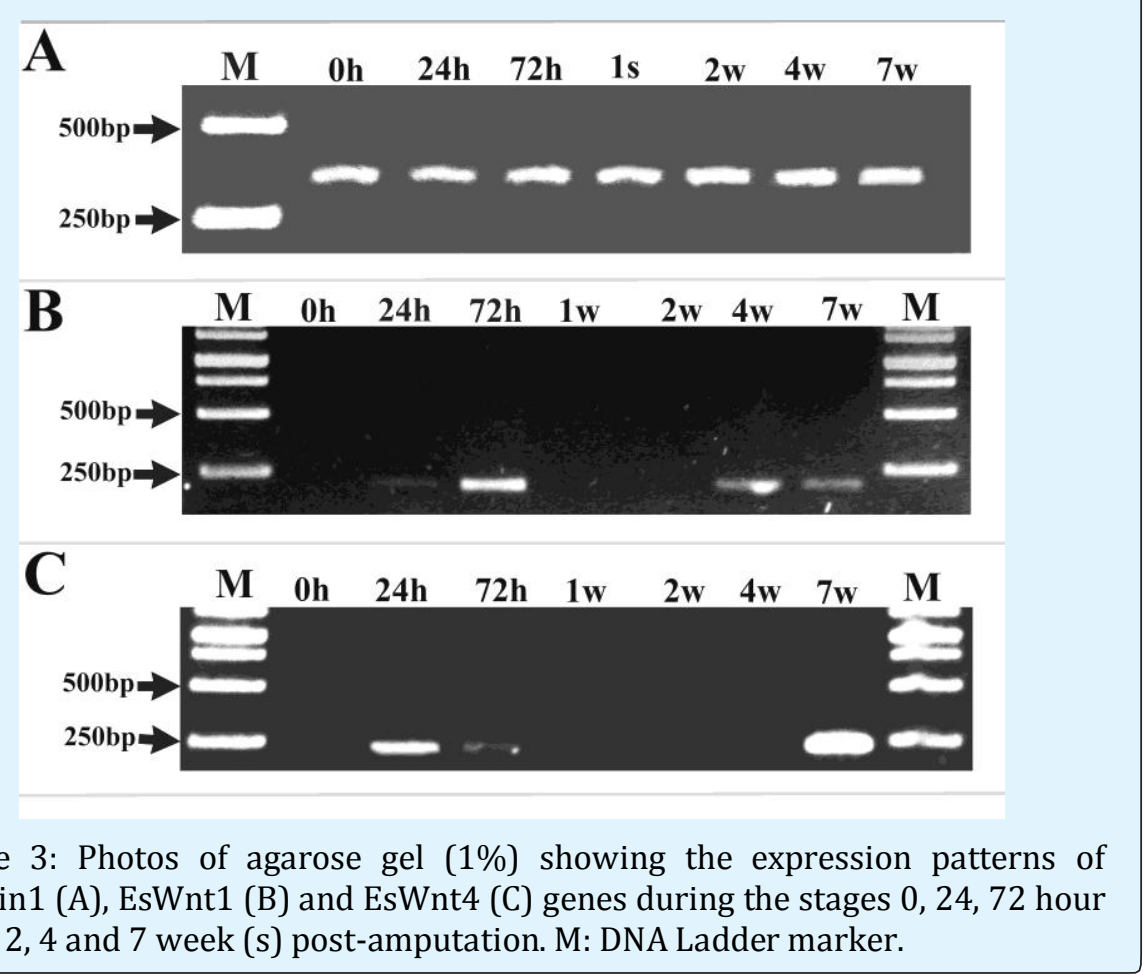

\section{Discussion}

The phylogenetic analysis showed that each identified Wnt gene in E. Sepositus (EsWnt1 and EsWnt4) was correctly clustered within its relevant Wnt group. Protein sequence analysis revealed that EsWnt1 shared high degree of similarity (99\%) with Wnt1 from other echinoderm species (the sea urchin S. purpuratus and the sea star Evasterias troschelii), and a significant degree of similarity (86\%) with Wnt1 gene for Homo sapiens. In a similar way the EsWnt4 gene produced a protein sequence that is almost identical to other Wnt4 genes described for two sea urchins (S. purpuratus and $P$. lividus). Most probably the evolutionarily conserved sequences of the different Wnt families are at the base of the well-know conservation of Wnt functions across the animal kingdom [28-31]. In fact it has been demonstrated that more than $85 \%$ of Wnt signalling pathway components were conserved between the sea urchin and human. Moreover, more than 55\% of Wnt proteins identified invertebrates have orthologs in echinoderms [32]. All these phylogenetic data suggest the possibility of making meaningful comparisons of the expression patterns of EsWnt1 and EsWnt4 genes in $E$. sepositus with those relatives in more distant taxa, namely vertebrates.

The temporal expression of EsWnt1 and EsWnt4 genes in the sea star E. sepositus at different stages of arm regeneration $(0,24,72$ hour (s), 1, 2, 3, 4 and 7 week (s) post-amputation), were studied using RT-PCR. The relative expression of the housekeeping gene Actin1 has been shown to remain constant over the period analysed; however, the expression profiles of EsWnt1 and EsWnt4 genes showed variation at the different stages of regeneration in E. sepositus.

An early expression of EsWnt1 and EsWnt4 genes has been noted at 24 and 72 hours post-amputation. Our previous histological studies suggest that these two genes are probably involved in the wound healing process and in the cell migration to the oedematous area. In fact, the activation of Wnt signalling pathway seems to be one of the initial molecular responses to the trauma in different species [21]. Strikingly in mammalian organs and tissues with limited regenerative capacity, Wnt signalling is still necessary for the repair phase. For instance, Wnt signalling is required for the regeneration of bone after skeletal fracture [33-35]. Also, the inhibition of Wnt signalling during skin wounding prevents formation of epithelial appendages including hair and sweat glands, which results in prominent scarring of the epidermis [36]. Also, if Wnt signalling is repressed the scarring of myocardial infarctions worsens with the result of myocardial rupture [37]. In planarians, it has been documented that Wnt signalling plays a crucial role in the regulation of the earliest events of regeneration that involve dedifferentiation and blastema morphogenesis [38]. It has been showed that wound healing participates directly in the regeneration polarity set up by inducing wntP-1 expression in Schmidtea mediterranea.WntP-1 was expressed at both anteriorand posterior-facing wounds rapidly after injury, which suggested that WntP-1 is required in controlling the regeneration polarity of the amputated parts [18]. 


\section{International Journal of Biochemistry \& Physiology}

The expression of Wnt1 in E. sepositus regenerating arm was up regulated at 24 hours pa stage, and then decreased slightly at the $3^{\text {rd }}$ day pa. In relation with our results, Hayashi, et al. [39] found that the expression of wnt1/P-1 in the planarian Dugesia japonica has two phases; a first phase at day 1 and a second phase after 3 days of amputation. Twenty-four hours post amputation Wnt1/P-1 expression was up regulated in the differentiated cells at the posterior end (stump) and, then at the $3^{\text {rd }}$ day, this gene showed a down-regulation in the blastema. These authors speculated that the wnt1/P-1 signalling in differentiated cells might be involved in the activation of the posterior genes in stem cell-derived cells (the first phase), these latter will migrate to the site of the lesion giving rise to the blastema (the second phase). Such model of the sequential posterior regeneration process could occur in the sea star E. sepositus arm regeneration [7,8]. It is possible that EsWnt1 is involved in setting up the polarity of the new structures in the regenerate. Indeed, the expression of EsWnt1, at 24 and 72 hours pa, coincides with the beginning of the migration and accumulation of recruited cells to the wound area [7]. It could very well be that EsWnt1 expression in the differentiated cells of the stump triggered cell dedifferentiation/migration. Then, the EsWnt1 expression in the accumulated cells of the oedematous area (3 days pa) will control the proximo-distal regeneration of the new arm-tip. In mammals, it has been shown that the expression of Wnt1 caused a dramatic increase in the proliferation of muscle satellite cells. In contrast, exposure of satellite cells to Wnt4 decreases proliferation [40]. We suggest that this could explain the inverse relationships between the expression pattern of EsWnt4 and EsWnt1genes in the repair phase ( 24 and 72 hours pa). It is possible that the dedifferentiation of damaged muscle in E. sepositus, which is probably activated by EsWnt1, is down regulated by EsWnt4.

A late expression phase of the EsWnt1 has been noted at 4 and 7 weeks pa. According to our histological studies, this expression concurs with the early regenerative phase (1-6 weeks pa), which is characterized by tissue rearrangement, morphogenetic processes and initial differentiation events (mainly neurogenesis and skeletogenesis) [8]. The EsWnt1gene might play roles in the morphogenesis of some missing tissues. Similarly, EsWnt4 showed a delayed expression (7 weeks pa). This expression happened temporarily together with the establishment of the new muscle bundles in the regenerating arm. Several studies demonstrated that Wnt4 gene acts as a stimulator of cell muscle proliferation and differentiation [41]. Indeed, it has been shown that the Wnt4 gene is involved in the renewal of muscles after trauma [42] contributing, later on, to the formation of muscle articulations during chick limb development [43]. In mammals, high levels of
Wnt4 expression have been observed in mice lacking myostatin; a growth factor that limits muscle tissue growth. Furthermore, it has been proved that Wnt4 expression stimulates the proliferation of muscle satellite cells in vitro [44]. In addition to its contribution in myogenesis, Wnt4 signalling enhances bone regeneration in craniofacial defects [45]. This gene can stimulate bone formation through several molecular mechanisms, including the renewal of stem cells, the stimulation of proliferation and/or differentiation of osteoblast and the inhibition of apoptosis [46,47]. In summary, it has been verified that Wnt4 regulates myogenesis and skeletogenesis. It is thus likely that EsWnt4 expression is involved in the formation of the new muscles, and, plausibly, in skeletogenesis during arm regeneration of E. sepositus.

All in all, here we have shown the preliminary characterization of two Wnt genes expressed during the regeneration of the sea stars arm tips. The thorough analysis of the expression domains should provide us with key insights on the processes that regulate the proliferation and differentiation of new tissues during regeneration.

\section{References}

1. Goss RJ (1992) The evolution of regeneration: adaptive or inherent? J Theor Biol 159(2): 241-260.

2. Horvat JC (2010) Paraplégie: Espoirs et réalités de la recherche fondamentale et clinique. Paris: Lavoisier S.A.S, pp : 180.

3. Candia Carnevali MD, Burighel P (2010) Regeneration in echinoderms and ascidians. ELS Wiley: Chichester.

4. Moss C, Hunter J, Thorndyke MC (1998) Pattern of bromodeoxyuridine incorporation and neuropeptide immunoreactivity during arm regeneration in the starfish Asterias rubens. Phil Trans R Soc London B Sci 353(1367): 421-436.

5. Mladenov PV, Bisgrove B, Aostra S, Burke RD (1989) Mechanisms of arm-tip regeneration in the sea star, Leptasterias hexactis. Roux's Arch Dev Biol 189(1): 19-28.

6. Ben Khadra Y, Said K, Thorndyke MC, Martinez P (2014) Homeobox Genes Expressed During Echinoderm Arm Regeneration. Biochem Genet 52(3-4): 166-180.

7. Ben Khadra Y, Ferrario C, Di Benedetto C, Said K, Bonasoro F, et al. (2015) Wound repair during arm regeneration in the red starfish Echinaster sepositus. Wound Repair Regen 23(4): 611-622. 


\section{International Journal of Biochemistry \& Physiology}

8. Ben Khadra Y, Ferrario C, Di Benedetto C, Said K, Bonasoro F, et al. (2015) Re-growth, morphogenesis, and differentiation during starfish arm regeneration. Wound Repair Regen 23(4): 623634.

9. Veraksa A, Campo MD, Mc Ginnis W (2000) Developmental patterning genes and their conserved functions: from model organisms to humans. Mol Gen Metab 69(2): 85-100.

10. Gehring WJ, Kloter U, Suga H (2009) Evolution of the Hox gene complex from an evolutionary ground state. Curr Top Dev Biol 88: 35-61.

11. Burke AC, Nelson CE, Morgan BA, Tabin C (1995) Hox genes and the evolution of vertebrate axial morphology. Development 121(2): 333-346.

12. Wodarz A, Nusse R (1998) Mechanisms of Wnt signaling in development. Annu Rev Cell Dev Biol 14: 59-88.

13. Niehrs C (2001) Developmental biology. Solving a sticky problem. Nature 413(6858): 787-788.

14. Veeman MT, Axelrod JD, Moon RT (2003) A second canon: functions and mechanisms of b-cateninindependent Wnt signaling. Dev Cell 5(3): 367-377.

15. Gurley KA, Rink JC, Sanchez Alvarado A (2008) Beta-catenin defines head versus tail identity during planarian regeneration and homeostasis. Science 319(5861): 323-327.

16. Petersen CP, Reddien PW (2008) Smedbetacatenin-1 is required for anteroposterior blastema polarity in planarian regeneration. Science 319(5861): 327-330.

17. Petersen CP, Reddien PW (2009) Wnt signaling and the polarity of the primary body axis. Cell 139(6): 1056-1068.

18. Petersen CP, Reddien PW A (2009) woundinducedWnt expression program controls planarian regeneration polarity. Proc Natl Acad Sci USA 106(40): 17061-17066.

19. Yazawa S, Umesono $Y$, Hayashi T, Tarui H, Agata K (2009) Planarian Hedgehog/Patched establishes anterior-posterior polarity by regulating Wnt signaling. Proc Natl Acad Sci USA 106(52): 2232922334.

20. Kawakami Y, Rodriguez-Esteban C, Raya M, Kawakami H, Marti M, et al. (2006) Wnt/betacatenin signaling regulates vertebrate limb regeneration. Genes Dev 20(23): 3232-3237.
21. Whyte JL, Smith AA, Helms JA (2012) Wnt Signaling and Injury Repair. Cold Spring Harb Perspect Biol 4(8): a008078.

22. Mashanov Vladimir S, Zueva OR, Garcia-Arraras JE (2012) Expression of Wnt9, TCTP, and Bmp1/Tll in sea cucumber visceral regeneration. Gene Expr Patterns 12(1-2): 24-35.

23. Ferkowicz MJ, Stander MC, Raff RA (1998) Phylogenetic relationships and developmental expression of three sea urchin Wnt genes. Mol Biol Evol 15(7): 809-819.

24. Benson DA, Karsch-Mizrachi I, Lipman DJ, Ostell J, Wheeler DL (2005) GenBank. Nucleic Acids Res 33: D34-D38.

25. Larkin MA, Blackshields G, Brown NP, Chenna R, McGettigan PA, et al. (2007) Clustal W and Clustal X version 2.0. Bioinformatics 23(21): 2947-2948.

26. Tamura K, Stecher G, Peterson D, Filipski A, Kumar S (2013) MEGA6: Molecular Evolutionary Genetics Analysis version 6.0. Mol Biol Evol 30(12): 27252729.

27. Nei M, Kumar S (2000) Molecular Evolution and Phylogenetics. Oxford University Press, New York.

28. Cadigan KM, Nusse R (1997) Wnt signaling: a common theme in animal development. Genes Dev 11(24): 3286-3305.

29. Croce JC, McClay DR (2006) The canonical Wnt pathway in embryonic axis polarity. Semin Cell Dev Biol 17(2): 168-174.

30. Klein TJ, Mlodzik M (2005) Planar cell polarization: an emerging model points in the right direction. Annu Rev Cell Dev Biol 21: 155-176.

31. Mlodzik M (2002) Planar cell polarization: do the same mechanisms regulate Drosophila tissue polarity and vertebrate gastrulation? Trends Genet 18(11): 564-571.

32. Croce JC, Wu SY, Byrum C, Xu Ronghui, Wikramanayake L, et al. (2006) A genome-wide survey of the evolutionarily conserved Wnt pathways in the sea urchin Strongylocentrotus purpuratus. Dev Biol 300(1): 121-131.

33. Chen Y, Whetstone HC, Lin AC, Nadesan P, Wei Q, et al. (2007) b-Catenin signaling plays a disparate role in different phases of fracture repair: Implications for therapy to improve bone healing. PLoS Med 4(7): 1216-1229. 


\section{International Journal of Biochemistry \& Physiology}

34. Leucht P, Kim JB, Helms JA (2008) b-Catenindependent Wnt signaling in mandibular bone regeneration. J Bone Joint Surg Am 90(1): 3-8.

35. Gurung A, Uddin F, Hill RP, Ferguson PC, Alman BA (2009) b-Catenin is a mediator of the response of fibroblasts to irradiation. Am J Pathol 174(1): 248255.

36. Ito M, Yang Z, Andl T, Cui C, Kim N, et al. (2007) Wnt-dependent de novo hair follicle regeneration in adult mouse skin after wounding. Nature 447(7142): 316-320.

37. Chen L, Wu Q, Guo F, Xia B, Zuo J (2004) Expression of Dishevelled-1 in wound healing after acute myocardial infarction: Possible involvement in myofibroblast proliferation and migration. J Cell Mol Med 8(2): 257-264.

38. Ghosh S, Roy S, Séguin Carl, Bryant SV, Gardiner DM (2008) Analysis of the expression and function of Wnt-5a and Wnt-5b in developing and regenerating axolotl (Ambystoma mexicanum) limbs. Develop Growth Differ 50(4): 289-297.

39. Hayashi T, Motoishi $M$, Yazawa S, Itomi $K$, Tanegashima C, et al. (2011) A LIM-homeobox gene is required for differentiation of Wnt-expressing cells at the posterior end of the planarian body. Development 138(17): 3679-3688.

40. Otto A, Schmidt C, Luke G, Allen S, Valasek P, et al. (2008) Canonical Wnt signalling induces satellitecell proliferation during adult skeletal muscle regeneration. J Cell Sci 121(17): 2939-2950.
41. Takata H, Terada $\mathrm{K}$, Oka $\mathrm{H}$, Sunada $\mathrm{Y}$, Moriguchi $\mathrm{T}$, et al. (2007) Involvement of Wnt4 signaling during myogenic proliferation and differentiation of skeletal muscle. Dev Dyn 236(10): 2800-2807.

42. Polesskaya A, Seale P, Rudnicki MA (2003) Wnt signaling induces the myogenic specification of resident $\mathrm{CD} 45+$ adult stem cells during muscle regeneration. Cell 113(7): 841-852.

43. Loganathan PG, Nimmagadda S, Huang R, Scaal M, Christ B (2005) Comparative analysis of the expression patterns of Wnts during chick limb development. Histochem Cell Biol 123(2): 195-201.

44. Bernardi H, Gay S, Fedon Y, Vernus B, Bonnieu A, et al. (2011) Wnt4 activates the canonical betacatenin pathway and regulates negatively myostatin: functional implication in myogenesis. Am J Physiol Cell Physiol 300(5): 1122-1138.

45. Chang J, Sonoyama W, Wang Z, Jin Q, Zhang C, et al. (2007) Noncanonical Wnt-4 Signaling Enhances Bone Regeneration of Mesenchymal Stem Cells in Craniofacial Defects through Activation. J Biol Chem 282(42): 30938-30948.

46. Kato M, Patel MS, Levasseur R, Lobov I, Chang BH, et al. (2002) Cbfa1-independent decrease in osteoblast proliferation, osteopenia, and persistent embryonic eye vascularization in mice deficient in Lrp5, a Wnt coreceptor. J Cell Biol 157(2): 303-314.

47. Holmen SL, Zylstra CR, Mukherjee A, Sigler RE, Faugere MC, et al. (2005) Essential role of betacatenin in postnatal bone acquisition. J Biol Chem 280(22): 21162-21168. 